\title{
High Serum CA19-9 Concentration Indicates High Chemosensitivity and Better Survival in Advanced Urothelial Carcinoma
}

\author{
HIROSHI YAEGASHI, KOUJI IZUMI, SUGURU KADOMOTO, RENATO NAITO, \\ TOMOYUKI MAKINO, HIROAKI IWAMOTO, TAKAHIRO NOHARA, \\ KAZUYOSHI SHIGEHARA, YOSHIFUMI KADONO and ATSUSHI MIZOKAMI \\ Department of Integrative Cancer Therapy and Urology, \\ Kanazawa University Graduate School of Medical Science, Kanazawa, Japan
}

\begin{abstract}
Background: The present study was conducted to investigate whether baseline serum carbohydrate antigen (CA) 19-9 predicts prognosis or treatment effect in patients with advanced urothelial carcinoma $(U C)$. Materials and Methods: We retrospectively analyzed data of patients diagnosed with locally advanced or metastatic or recurrent UC between April 2008 and November 2014. CA19-9 was determined using enzyme-linked immunosorbent assay (ELISA) and the relationship between CA19-9 and prognosis were analyzed. Results: Of 40 patients, seven with CA19-9 $\leq 2 \mathrm{U} / \mathrm{ml}$ who were suspected of having Lewis A-negative blood type and two patients with advanced metastatic liver disease were excluded. UC-specific survival in metastatic disease significantly correlated with prognosis $(p=0.018)$. Overall survival in patients with high serum CA19-9 demonstrated a significantly better prognosis than in those with low concentrations (log-rank test, $p=0.032)$. Conclusion: High baseline serum CA19-9 may predict a good prognosis in patients with advanced UC.
\end{abstract}

According to recent Surveillance, Epidemiology, and End Results (SEER) program data for the United States, over the past decade, the incidence of new bladder cancer has been decreasing on an average of $1 \%$ each year and mortality due to bladder cancer has not changed (1). However, in Japan, both the incidence of and mortality due to urothelial carcinoma (UC) (i.e. renal pelvic, ureteral, and bladder

Correspondence to: Dr. Kouji Izumi, Department of Integrative Cancer Therapy and Urology, Kanazawa University Graduate School of Medical Science, 13-1 Takaramachi, Kanazawa, Ishikawa 920-8640, Japan. Tel: +81 762652393, Fax: +81 762344263, e-mail: azuizu2003@yahoo.co.jp

Key Words: CA19-9, advanced urothelial carcinoma, renal pelvis, ureter, bladder. cancer) have been increasing steadily over the course of the past year (2). There is an urgent need to improve the cure rate for UC, particularly advanced UC, for which predictive markers for diagnosis or treatment effect are lacking.

Carbohydrate antigen (CA) 19-9 has recently been established as a tumor marker in cancers including pancreatic, gallbladder, and colon cancer. Several recent reports have suggested that a high CA19-9 concentration is a poor prognostic factor $(3,4)$. Therefore, we examined the relationship between serum CA19-9 concentrations and background factors, including clinical or pathological stage, to determine whether serum CA19-9 concentration is useful in predicting prognosis or treatment effect in patients diagnosed with advanced UC.

\section{Materials and Methods}

The medical records of patients diagnosed with locally advanced or metastatic or recurrent UC in our Department between April 2008 and November 2014 were evaluated retrospectively. The Ethics Committee of Kanazawa University Graduate School of Medical Science approved the present study (approval number 1805). Pathological TNM stage was recorded for patients who underwent radical surgery and clinical TNM stage for those who did not. Metastatic disease was defined as the presence of extra-regional lymph node or other organ metastases. CA19-9 concentration was determined using enzyme-linked immunosorbent assay for all cases and was expressed in U/mI. In each case, serum CA19-9 concentration was measured at least once at baseline, prior to induction of any therapy. If serum CA19-9 concentration was elevated, it was re-measured several times at the physician's discretion. Patients with another type of cancer (lung, pancreatic, colon, gallbladder, bile duct, gynecological, or breast cancer) producing an elevated CA19-9 concentration were excluded. GraphPad Prism 7 software (GraphPad Software Inc., San Diego, CA, USA) was used to analyze data and obtain figures. One-way analysis of variance (ANOVA) and Student's or Welch's $t$-test were used for comparative data analysis. Kaplan-Meier curves were used to compare survival times. A $p$-value of less than 0.05 was considered statistically significant for each analysis. 
Table I. Characteristics of patients.

\begin{tabular}{lc}
\hline Characteristic & $\mathrm{n}=40$ \\
\hline Median age, years & \\
At diagnosis (or at first visit) & 75 \\
Gender & 28 \\
Male & 12 \\
Female & \\
Type of UC & 26 \\
Bladder & 6 \\
Ureter & 8 \\
Renal pelvis & \\
Resection of primary disease & 12 \\
Yes & 28 \\
No & \\
Type of anticancer chemotherapy & 14 \\
GEM+CDDP & 11 \\
M-VAC & 8 \\
GEM+CBDCA & 1 \\
PTX+CDDP+GEM & $5(3)$ \\
Arterial infusion chemotherapy (with EBRT) & \\
\hline
\end{tabular}

GEM: Gemcitabine, CDDP: cisplatin, M-VAC: methotrexate, vincristine, adriamycin, cisplatin, CBDCA: carboplatin, PTX: paclitaxel, EBRT: external beam radiation therapy.

Table II. Correlation of factors with serum cancer antigen (CA) 19-9 value.

\begin{tabular}{lccc}
\hline & $\begin{array}{c}\text { No. of } \\
\text { patients }\end{array}$ & $\begin{array}{c}\text { Median CA19-9 } \\
\text { (range), U/ml }\end{array}$ & p-Value \\
\hline Total & 31 & $16(5-167)$ & - \\
Type of UC & & $13(6-58)$ & Reference \\
$\quad$ Bladder & 18 & $22.5(6-140)$ & 0.096 \\
$\quad$ Ureter & 6 & $34(5-167)$ & \\
$\quad$ Renal pelvis & 7 & & Reference \\
T stage & 1 & 26 & \\
Tis & 1 & 10 & 0.034 \\
Ta & 3 & $9(6-32)$ & - \\
T1 & 6 & $13.5(6-34)$ & \\
T2 & 12 & $28.5(6-167)$ & \\
T3 & 7 & $15(12-64)$ & Reference \\
T4 & 1 & 5 & 0.276 \\
$\quad$ TX & & & \\
Tumor spread & 19 & $16(5-167)$ & \\
Non-metastatic & 12 & $14.5(6-64)$ & \\
Metastatic* & & & \\
\hline
\end{tabular}

UC: Urothelial cancer. *Metastatic disease included distant metastasis only.

\section{Results}

Overall, 40 patients were included in the analysis [age at diagnosis 57-86 years (median, 75 years); 28 male, 12 female] of whom 26 had bladder cancer, eight had renal pelvic cancer, and six had ureteral cancer. Table I shows
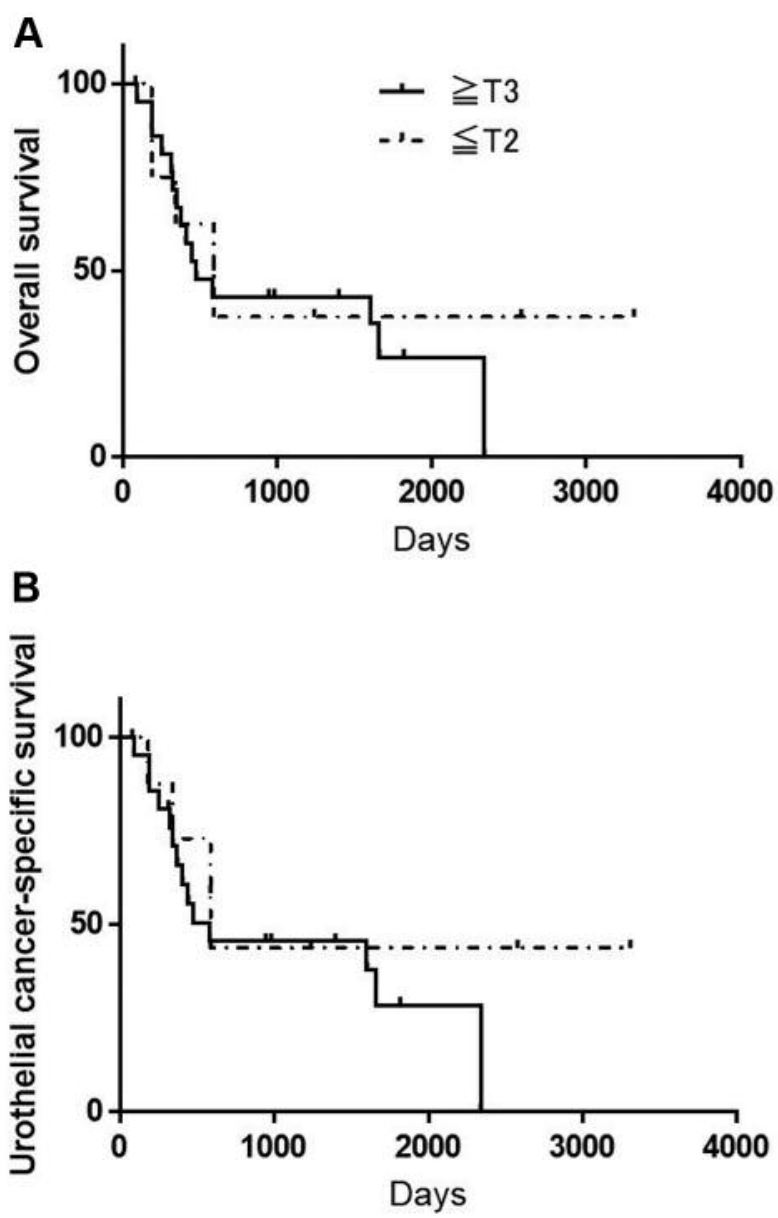

Figure 1. Kaplan-Meier analysis of overall survival (OS) (A) and urothelial cancer-specific survival (B) according to T-stage. T-Stage was not statistically significantly correlated with OS (log-rank test, $p=0.599$ ) nor urothelial cancer-specific survival (log-rank test, $p=0.423$ ).

patient demographics, clinical history, surgical history, and cancer chemotherapy $\operatorname{drug}(\mathrm{s})$ and route of administration. One patient had both renal pelvic and bladder cancer and one developed renal pelvic cancer following radical cystectomy for advanced bladder cancer. Furthermore, 12 cases underwent resection of primary disease. A baseline CA19-9 concentration of $30 \mathrm{U} / \mathrm{mI}$ was established as the upper cutoff value of normal. Seven patients who had a serum CA199 concentration of $\leq 2 \mathrm{U} / \mathrm{ml}$ at baseline were excluded following high suspicion for Lewis A-negative blood type [Le $(\mathrm{a}-\mathrm{b}-)$ or $\mathrm{Le}(\mathrm{a}-\mathrm{b}+)]$. Two patients with advanced disease who had extremely high serum CA19-9 concentration and multiple liver metastases were also excluded. For the remaining 31 patients, follow-up duration ranged from 82 to 3,309 days (median $=477$ days). The mean CA19-9 concentration was $30.3 \mathrm{U} / \mathrm{ml}$ (range $=5-167 \mathrm{U} / \mathrm{ml}$ ). Serum CA19-9 concentrations did not differ significantly among the 


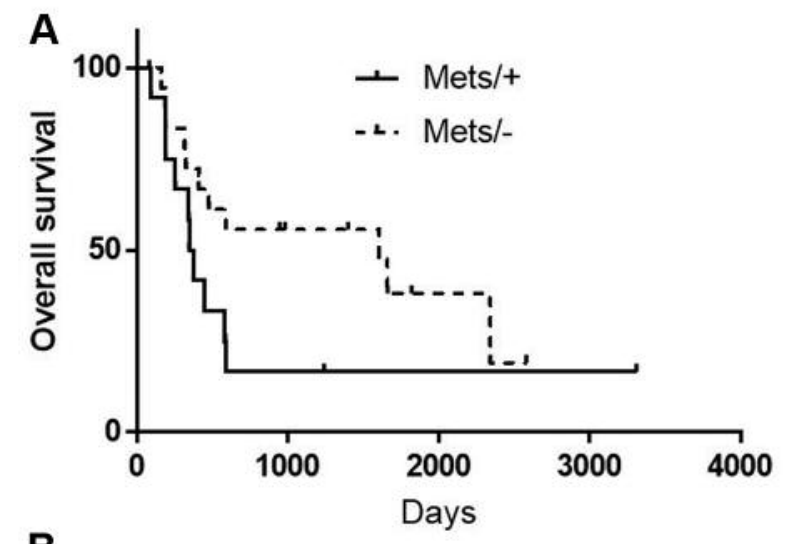

B

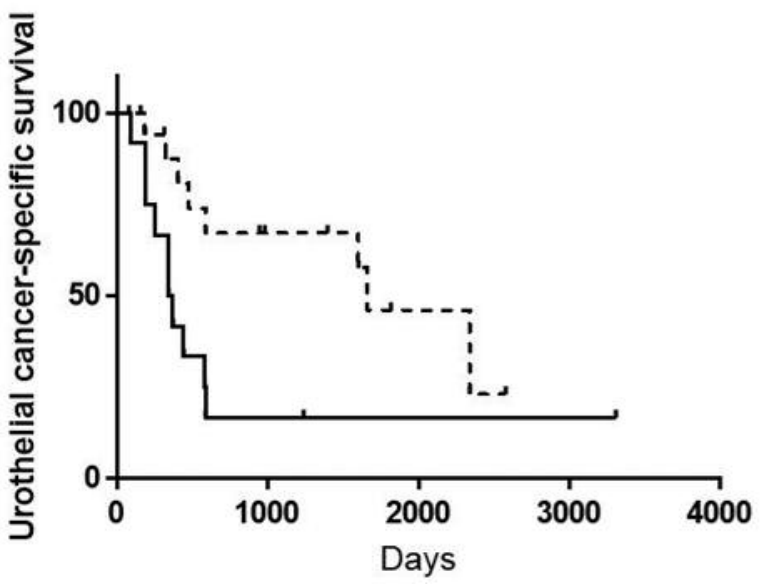

Figure 2. Kaplan-Meier analysis of overall survival (OS) (A) and urothelial cancer-specific survival (B) according to metastasis. Metastasis was not statistically significantly correlated with OS (logrank test, $p=0.103)$. Metastasis was statistically significantly associated with worse urothelial cancer-specific survival (log-rank test, $p=0.018$ ).

three types of UC (bladder, renal pelvic, and ureteral cancer). The increase in CA19-9 concentration was significantly higher for those with $\mathrm{T} 3$ or higher tumors than for T2 or lower tumors $(p=0.034)$, and did not differ significantly between those with metastatic and those with non-metastatic disease $(p=0.276)$ (Table II).

The interval between time of diagnosis and time of measurement of serum CA19-9 concentration did not differ significantly between those who had high CA19-9 level $(\geq 30 \mathrm{U} / \mathrm{ml}$ ) (one-way ANOVA $t$-test, $p=0.670$ ). Neither T-stage nor metastatic state correlated with prognosis, except with respect to UC-specific survival in metastatic disease $(p=0.018)$ (Figures 1 and 2). However, in terms of overall survival, a significantly better prognosis was seen in patients with a high serum CA19-9 concentration than in those with a low concentration (log-rank test, $p=0.032$ ) (Figure 3A),
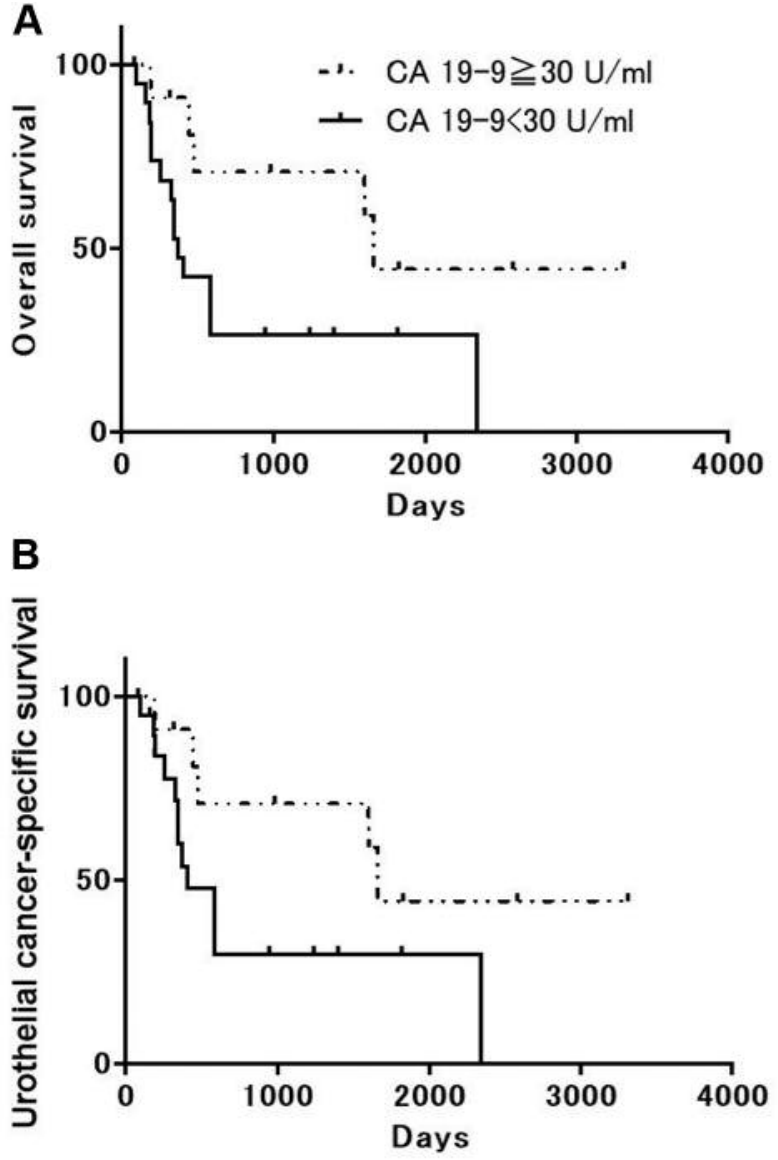

Figure 3. Kaplan-Meier analysis of overall survival (OS) (A) and urothelial cancer-specific survival $(B)$ according to serum carbohydrate antigen (CA) 19-9 level. High serum CA19-9 level was statistically significantly associated with better OS (log-rank test, $p=0.032$ ) but only marginally correlated with better urothelial cancer-specific survival (log-rank test, $p=0.062$ ).

and marginally significant in UC-specific survival (log-rank test, $p=0.062$ ) (Figure 3B).

\section{Discussion}

The tumor marker CA19-9, which recognizes the sialylated Lea carbohydrate structure (5), has primarily been used for serological diagnosis and follow-up of gastrointestinal and pancreatic malignancies (6-9). The interpretation of CA19-9 can be challenging because approximately $10 \%$ of the population are Lewis-negative and have undetectable CA199 concentrations regardless of tumor burden (6). Moreover, the expression of Lewis blood group antigens has been demonstrated to be affected by the presence of tumors in patients with cancer (10). Furthermore, a low serum CA199 concentration at baseline may not be useful for predicting 


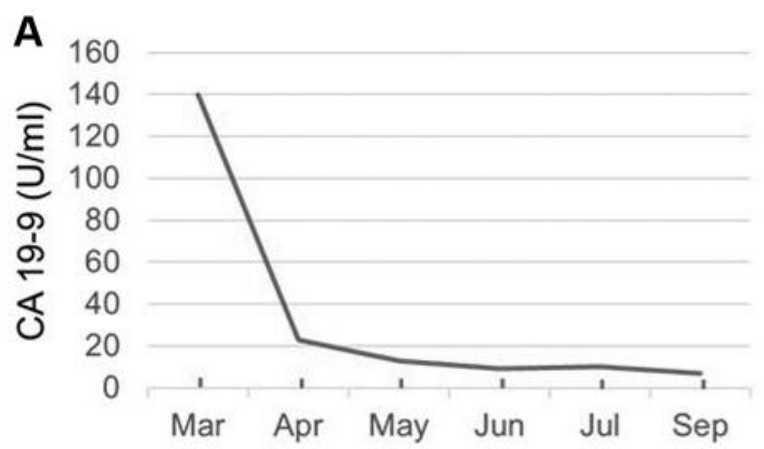

B

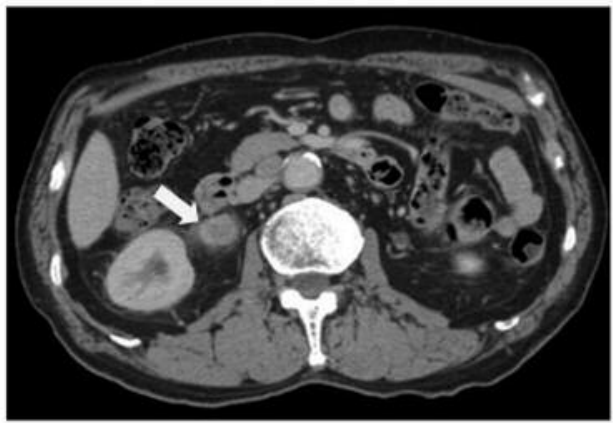

C

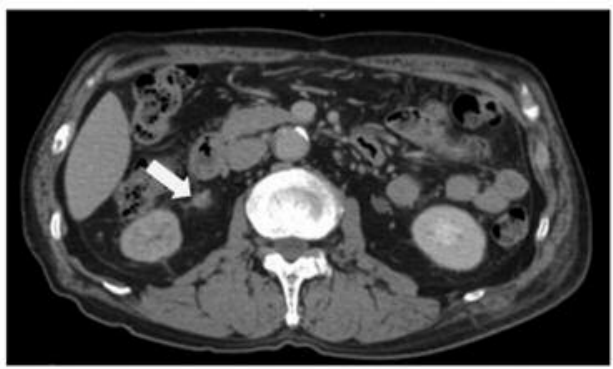

Figure 4. Case 1: A 71-year-old male, with right-sided ureteral cancer, cT3N2MO. A: Graph of the time course of carbohydrate antigen (CA) 19-9 value. B: Primary disease before anticancer chemotherapy (yellow arrow). C: Primary disease after anticancer chemotherapy (yellow arrow). Both CA19-9 value and radiological findings showed improvement.

prognosis or treatment effect in patients with advanced UC.

Suzuki et al. have described two mechanisms for the elevation of serum CA19-9 concentration in patients with advanced UC: One wherein the tumor itself produces CA199 and the other wherein a high renal pelvic pressure induces serum CA19-9 elevation because normal renal pelvic mucosa also produces CA19-9 (11).

In the present investigation, seven out of 11 cases with high CA19-9 concentration had hydronephrosis at initial diagnosis. Therefore, these seven cases may reflect serum CA19-9 elevation due to hydronephrosis.

The better prognosis of patients with high serum CA19-9 concentration might be explained by the fact that in three out of the 11 cases, serum CA19-9 was markedly reduced by the

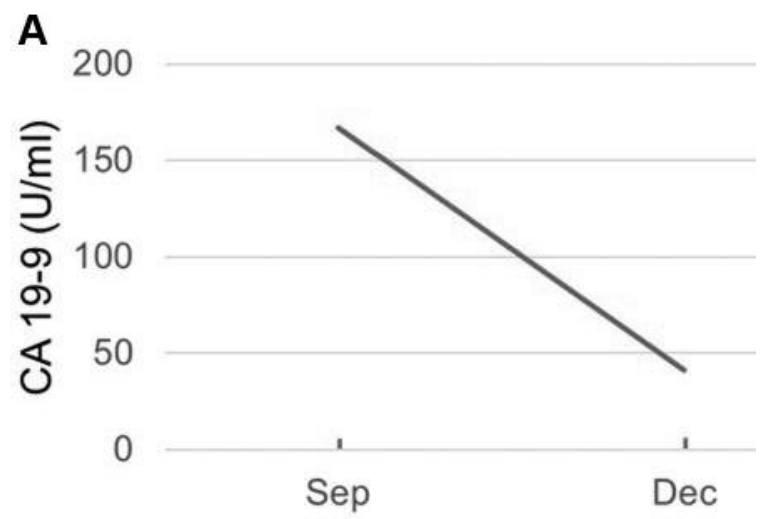

B

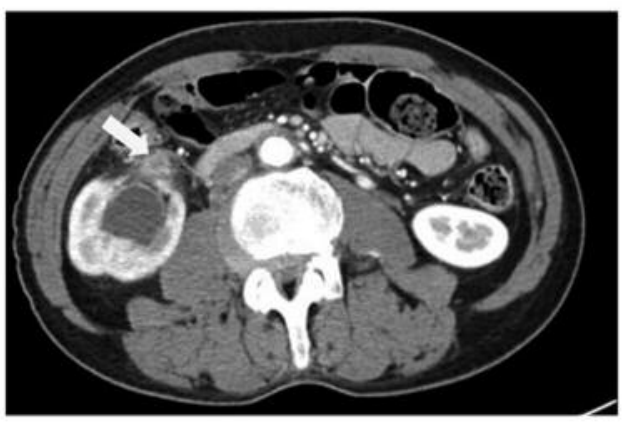

C

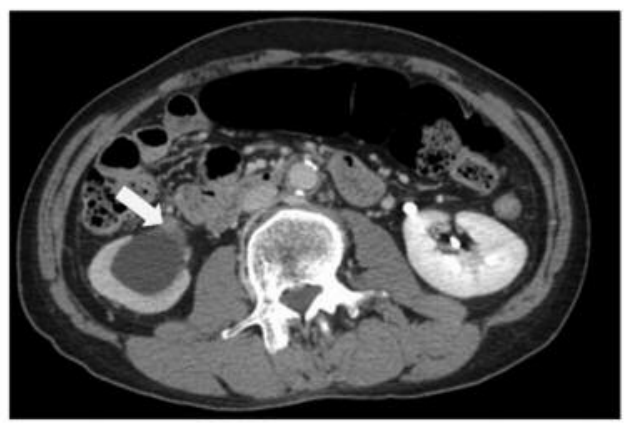

Figure 5. Case 2: A 58-year-old male, with right-sided renal pelvic cancer, cT3N2MO. A: Graph of the time course of CA19-9 value. B: Primary disease before anticancer chemotherapy (yellow arrow). $C$ : Primary disease after anticancer chemotherapy (a yellow arrow). Both CA19-9 value and radiological findings showed improvement.

induction of cancer chemotherapy (Figures 4-6). In these three cases, we considered the decrease in serum CA19-9 concentration to reflect radiological improvement.

Kanai $e t a l$. presented the case of a Japanese patient with ureteral UC having an extremely high serum CA19-9 concentration. Marked regression of the tumor was identified by radiological findings, and serum CA19-9 concentration returned to the normal range after three courses of methotrexate, vinblastine, doxorubicin, and cisplatin (M-VAC) therapy, following nephroureterectomy and partial cystectomy. 
A

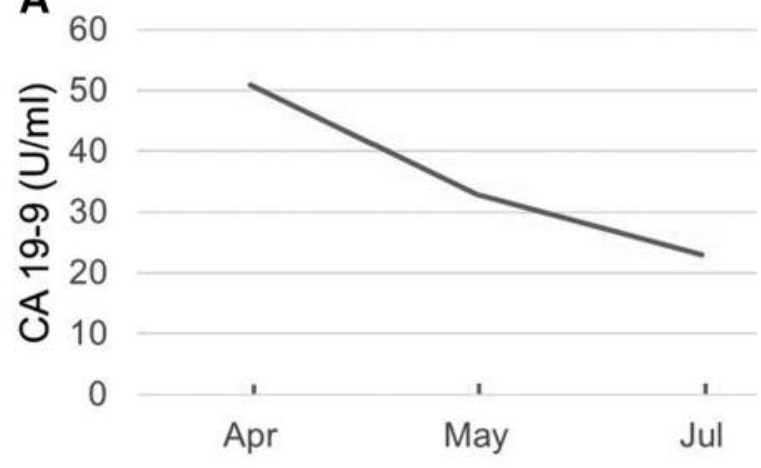

B

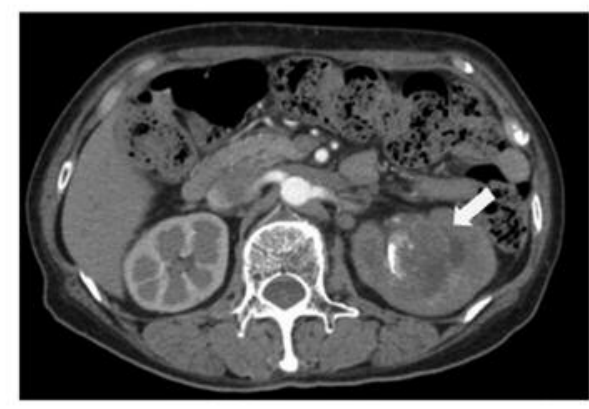

c

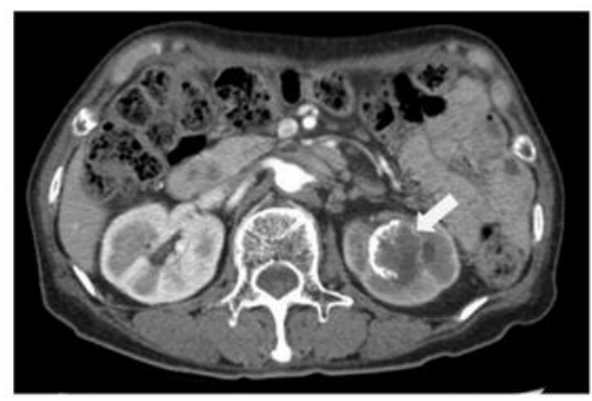

Figure 6. Case 3: A 74-year-old female, with left-sided renal pelvic cancer, cT3N1MO. A: Graph of the time course of CA19-9 value. B: Primary disease before anticancer chemotherapy (yellow arrow). C) Primary disease after anticancer chemotherapy (yellow arrow). Both CA19-9 value and radiological findings showed improvement.
At 6 months after resection, the patient was without evidence of local recurrence or metastasis (12). Another case of advanced bladder UC with high serum CA19-9 concentration reported by Inai et al. demonstrated an improvement of both CA19-9 concentration and radiological findings following arterial injection chemotherapy and radiotherapy (13). Both cases illustrate that patients with advanced UC having high serum CA19-9 concentration at baseline have high chemosensitivity, and therefore, serum CA19-9 would be a marker reflecting treatment effect (Figure 7).

The present study has some limitations. Firstly, this was a retrospective cohort with a small sample size and selection bias. However, previous reports and the present study suggest that serum CA19-9 concentration may be a predictor of prognosis and treatment effect, particularly in patients with advanced UC.

In conclusion, patients with advanced UC having high serum CA19-9 concentration at baseline have a high chemosensitivity. In these patients, the tumor marker CA19-9 may reflect response to therapy. Further investigation is warranted.

\section{Acknowledgements}

The Authors are grateful to Dr. Koushiro Ohtsubo, Division of Medical Oncology Cancer Research Insutitute, Kanazawa University, for helpful discussion.

\section{References}

1 Noone AM, Howlader N, Krapcho M, Miller D, Brest A, Yu M, Ruhl J, Tatalovich Z, Mariotto A, Lewis DR, Chen HS, Feuer EJ and Cronin KA (eds): SEER Cancer Statistics Review, 19752015, National Cancer Institute. Bethesda, MD (online). Available: https://seer.cancer.gov/csr/1975_2015/. Accessed October 31, 2018.

2 Hori M, Matsuda T, Shibata A, Katanoda K, Sobue T and Nishimoto H: Cancer incidence and incidence rates in Japan in 2009: A study of 32 population-based cancer registries for the Monitoring of Cancer Incidence in Japan (MCIJ) project. Jpn J Clin Oncol 45: 884-891, 2015.

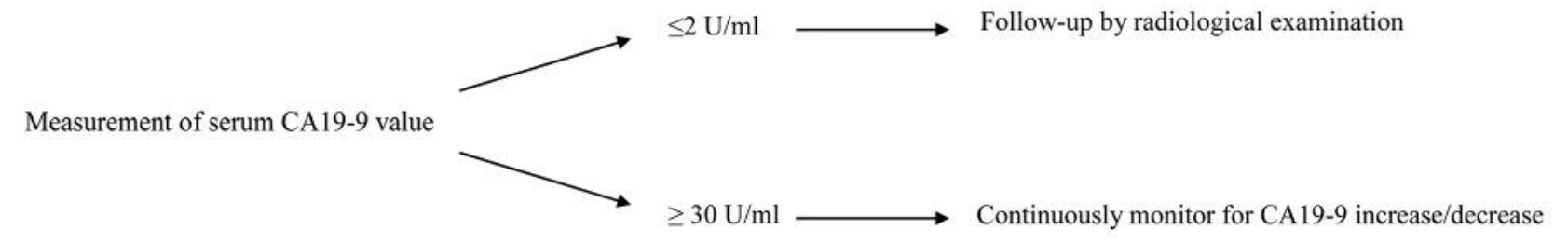

Figure 7. Scheme of measurement of serum carbohydrate antigen (CA) 19-9 value and evaluation of advanced urothelial cancer. 
3 Hegele A, Mecklenburg V, Varga Z, Olbert P, Hofmann R and Barth P: CA19.9 and CEA in transitional cell carcinoma of the bladder: Serological and immunohistochemical findings. Anticancer Res 30: 5195-5200, 2010.

4 Jha DK, Mittal A, Gupta SP and Sathian B: Significance of CA19-9 in predicting the prognosis of urothelial carcinoma: A hospital-based study from Nepal. Asian Pac J Cancer Prev 14: 4067-4069, 2013.

5 Scarà S, Bottoni $\mathrm{P}$ and Scatena R: CA 19-9: Biochemical and clinical aspects. Adv Exp Med Biol 867: 247-260, 2015.

6 Parra-Robert M, Santos VM, Canis SM, Pla XF, Fradera JMA and Porto RM: Relationship between CA 19.9 and the Lewis phenotype: Options to improve diagnostic efficiency. Anticancer Res 38: 5883-5888, 2018.

7 Ko AH, Hwang J, Venook AP, Abbruzzese JL, Bergsland EK and Tempero MA: Prognostic role of carcinoembryonic antigen and carbohydrate antigen 19-9 in metastatic colorectal cancer: A BRAF-mutant subset with high CA19-9 level and poor outcome. Serum CA19-9 response as a surrogate for clinical outcome in patients receiving fixed-dose rate gemcitabine for advanced pancreatic cancer. Br J Cancer 93: 195-199, 2005.

8 Thomsen M, Skovlund E, Sorbye H, Bolstad N, Nustad KJ, Glimelius B, Pfeiffer P, Kure EH, Johansen JS, Tveit KM, Christoffersen $\mathrm{T}$ and Guren TK: Prognostic role of carcinoembryonic antigen and carbohydrate antigen 19-9 in metastatic colorectal cancer: A BRAF-mutant subset with high CA 19-9 level and poor outcome. Br J Cancer 118: 1609-1616, 2018.
9 Nishio K, Kimura K, Amano R, Yamazoe S, Ohrira G, Nakata B, Hirakawa K and Ohira M: Preoperative predictors for early recurrence of resectable pancreatic cancer. World J Surg Oncol 15: 16, 2017.

10 Hamada E, Taniguchi T, Baba S and Maekawa M: Investigation of unexpected serum CA19-9 elevation in Lewis-negative cancer patients. Ann Clin Biochem 49: 266-72, 2012.

11 Suzuki K, Kumamaru T, Shioji Y, Morita T, Tokue A and Osawa $\mathrm{T}$ : Renal pelvic and ureteral cancer with extremely high serum level of carbohydrate antigen 19-9 (CA19-9): A case report. Nishinihon J Urol 62: 659, 2000.

12 Kanai S and Takagi Y: A case of CA19-9 producing transitional cell carcinoma of the ureter effectively responsive to combination chemotherapy. Hinyokika Kiyo 38: 1253-1256, 1992.

13 Inai $\mathrm{H}$, Shimazui $\mathrm{T}$, Yamamoto T, Yamauchi A, Uchida K, Takeshima $\mathrm{H}$ and Akaza $\mathrm{H}$ : A case of transitional cell carcinoma of the urinary bladder with high serum level of CEA and CA199. Hinyokika Kiyo 47: 583-586, 2001.

Received November 5, 2018

Revised November 29, 2018

Accepted December 4, 2018 